\title{
Discrete numerical approach to the Fredholm Integral Method for evaluating scattering by irregular dielectric particles
}

Article

Accepted Version

Ngobigha, F., Bebbington, D. and Carrea, L. (2017) Discrete numerical approach to the Fredholm Integral Method for evaluating scattering by irregular dielectric particles. IEEE Transaction on Antennas and Propagation, 65 (11). pp. 59495959. ISSN 1558-2221 doi:

https://doi.org/10.1109/TAP.2017.2751649 Available at https://centaur.reading.ac.uk/73014/

It is advisable to refer to the publisher's version if you intend to cite from the work. See Guidance on citing.

Published version at: http://ieeexplore.ieee.org/document/8036266/

To link to this article DOI: http://dx.doi.org/10.1109/TAP.2017.2751649

Publisher: IEEE

All outputs in CentAUR are protected by Intellectual Property Rights law, including copyright law. Copyright and IPR is retained by the creators or other copyright holders. Terms and conditions for use of this material are defined in the End User Agreement. 


\section{CentAUR}

Central Archive at the University of Reading

Reading's research outputs online 


\title{
Discrete numerical approach to the Fredholm Integral Method for evaluating scattering by irregular dielectric particles
}

\author{
Felix Ngobigha, David Bebbington, and Laura Carrea
}

\begin{abstract}
A new approach to the implementation of the Fredholm Integral Method (FIM) was developed to evaluate scattering by irregular dielectric particles. In this study, particles are modelled discretizing their volume with cells according to their weighted contents. The approach to FIM presented in this paper represents a departure from earlier work where the numerical integration is no longer based on expansion in a set of polynomials but on direct spatial integration. This approach which still involves contour integration method uses quandrantal contour in combination with a conditioning weighting function to control the magnitude of the integrand due to the power of the radial variable in the integrand being odd. The strength of our approach lies on the fact that computations are performed in the spatial frequency domain. As a result, the angular scattering pattern is strongly connected to the Spatial Fourier Transform of the scatterer; hence, for electrically small particles the angular spectrum is relatively smooth and the number of pivots required for integration is relatively low. This technique is well suited to the treatment of scattering from irregular inhomogeneous dielectric particles since only the distribution in space of the dielectric constants needs to be defined. Numerical results also confirm the inadequacy of effective medium theories in evaluating scattering characteristics of inhomogeneous particles.
\end{abstract}

Index Terms-Born terms, inhomogeneous medium, effective medium thoeries, discretization.

\section{INTRODUCTION}

$\mathbf{T}$ he melting layer is a region where the transition from ice crystals to rain drops happens. At this layer, particles are mainly melting ice crystals, a mixture of ice, air and water. This region has been widely studied since it produces high values in radar reflectivity imaging causing potentially an overestimation of precipitation [1], [2] and [3]. Simulations of radar signature at the melting layer includes a particle scattering model of melting snow [4], [5].

Evaluating the scattering characteristics of these particles is quite difficult due to change in their geometrical and electrical properties [5]. The complex nature of the melting process and the lack of accurate experimental/measured data of the effective dielectric constant of the melting snow flakes lead to relying mainly on two particle melting models as discussed in [1]. Mixing theories [6], [7] offer methods to estimate the effective dielectric constant of mixed phase hydrometeors. It has been suggested that in this case, the estimation of their

F. Ngobigha and D. Bebbington are with the School of Computer Science and Electronic Engineering, University of Essex, Colchester, CO4 3SQ, U.K. E-mail: fngobi@essex.ac.uk, david@essex.ac.uk.

L. Carrea is with the Department of Meteorology, University of Reading, Reading, RG6 6AH, U.K. E-mail: 1.carrea@reading.ac.uk. scattering characteristics could be done by computing the effective dielectric constant, averaging the optical properties of an equivalent materials (two-part effective medium mixing formula) [6] and subsequently evaluating the scattering characteristics of homogeneous particle. However, it has been shown in [8], [9], [10], [11], [12] that this approach is not fully consistent with the complex nature of the inhomogeneous scatterer. Moreover, there is no known unique effective medium mixing theory for three or more dielectric components. Although our approach has been developed to compute microwave scattering from mixed phase irregular hydrometeors in the melting layer, its formulation is quite general and can be applied to other areas such as for biological blood cells [13] or for small chemical particles [14]. To address this problem fully, we propose to model a generally discretised particle. This address the irregular shape of the scatterer and ultimately can cope with inhomogeneities of the dielectric particle.

Several techniques are available for the evaluation of microwave scattering by irregular dielectric particles. Among them, there are the Mie theory [15], [16], the Discrete Dipole Approximation (DDA) [17], [18], and Fredholm Integral Method [19], [20], [21]. The DDA is based on replacing the scattering particle with interacting dipoles. This approach has shortcomings associated with the size of matrix equations to be solved if the particle is electrically large (defined as a particle of refractive index far greater than 1 or the particle size is relatively large compared with the wavelength. In the text we use parameter $x=k a$ where $k$ is $\frac{2 \pi}{\lambda}$ and $a$ is the equivalent volume radius of the particle) and the problem becomes computationally difficult.

The FIM approach of Holt [19] was formulated in terms of a volume integral equation. Generally it can be solved with an expansion in a set of polynomials and then applying a series of iterative approximations known as the Born approximations. In general, there is a problem with this since it is well known [22], [23] that for large dielectric constant the Born series do not converge. It is noteworthy to mention that the method is not a Born-series which does not necessarily converge. The method is exact if the second order term can be exactly computed. In [19], an exact integral equation approach involving only the first and second Born terms was proposed. However, as also stated by the authors, the main limitation is the evaluation of the second Born term which for large particles requires too large machine storage and/or computation time. Moreover, their approach has not been tested 
for inhomogeneous scatterers but they envisage difficulties in the evaluation of the second Born term. Consequently, these techniques are not fully satisfactory to evaluate the scattering properties of irregular hydrometeors.

In the present work, a new perspective is proposed. It is still based on the central equations of the FIM introduced in [19] for scattering by dielectric homogeneous particles but in contrast with their approach, in case of using an expansion in a set of polynomials we apply a direct spatial integration. In this way, our approach retains the property of having second order accuracy owing to the variational properties of the integral. The scatterer is modelled within a finite regular lattice field of cubic or spherical cells. The first and second Born terms are evaluated for a cell at the origin, while the contributions of all other cells are evaluated efficiently using the Fourier Shift Theorem. We have named our numerical approach to the Fredholm Integral Method as the Discrete Method (DM) and it was pioneered in [24]. The DM allows to explicitly evaluate scattering amplitude functions of irregular hydrometeors avoiding any of the established effective medium theories or empirical methods. The approach is computational efficient and the complexity of implementation is low. In particular, in terms of the RAM resources the storage goes as the square of discretization scale compared to the DDA. Regarding the computational complexity, the second Born term is dominant and its complexity is $O\left(N^{2}\right)$ where $N$ is number of elements, while for the DDA matrix inversion is $O\left(N^{3}\right)$. There is some evidence that iterative methods in DDA can converge much faster than full matrix inversion, but this is not formally guaranteed. In case of thousands of hydrometeors, variation of the scatterers orientation is trivial and previous work [19] established that it is feasible to select a relatively small number of size over a certain range, and to implicitly interpolate when computing integrals.

The organization of the paper is as follows. In section II the derivation of the Discrete Method is presented. In section III we validate our approach comparing the results for spherical homogeneous particles with the Mie theory. Finally, in section IV we evaluate scattering properties of spherical mixed phase hydrometeors using the Discrete Method with and without mixing theories. Important conclusions on the validity and limitations of effective mixing theories are drawn.

\section{Discrete Method Formulation}

The derivation of the Discrete Method equations for evaluating the electromagnetic waves scattering by homogeneous particles is presented. The extension to inhomogeneous particles such as melting irregular hydrometeors is straightforward. The notation of [19] is used for the formulation.

The Maxwell's differential equation valid inside and outside of the volume containing a dielectric medium can be written as [25]:

$$
\nabla \times(\nabla \times \mathbf{E})-k_{0}^{2} \mathbf{E}=k_{0}^{2}\left(n^{2}-1\right) \mathbf{E},
$$

where $\mathbf{E}$ is the electric field, $k_{0}$ the free space wavenumber and $n$ the refraction index of the dielectric medium. In (1) we assume a $e^{-i \omega t}$ time dependence at angular frequency $\omega$ and for simplicity we suppress the time factor in all the fields throughout the paper.

The general solution of the inhomogeneous linear differential equation (1) is the sum of the complementary solution of the correspondent homogeneous equation and the particular solution of the inhomogeneous equation. Thus, the homogeneous equation is

$$
\nabla \times \nabla \times \mathbf{E}-k_{0}^{2} \mathbf{E}=0,
$$

which describes the field in the absence of the scatterer. The appropriate physical particular solution of equation (1) must satisfy the scattered field generated by the forcing function. Using the Green's function method to evaluate the scattered field $\mathbf{E}$, the free space dyadic Green's function $\overline{\mathbf{G}}\left(\mathbf{r}, \mathbf{r}^{\prime}\right)$ associated with the inhomogeneous equation (1) satisfies the differential equation [26]

$$
\nabla \times \nabla \times \overline{\mathbf{G}}\left(\mathbf{r}, \mathbf{r}^{\prime}\right)-k_{0}^{2} \overline{\mathbf{G}}\left(\mathbf{r}, \mathbf{r}^{\prime}\right)=-\overline{\mathbf{I}} \delta\left(\mathbf{r}, \mathbf{r}^{\prime}\right),
$$

where $\overline{\mathbf{I}}$ is the unit dyadic. The solution of equation (3) can be expressed as in [27] and [28]:

$$
\overline{\mathbf{G}}\left(\mathbf{r}, \mathbf{r}^{\prime}\right)=\left(\overline{\mathbf{I}}+k_{0}^{-2} \nabla \nabla\right) G\left(\mathbf{r}, \mathbf{r}^{\prime}\right),
$$

where $G\left(\mathbf{r}, \mathbf{r}^{\prime}\right)=\exp \left(\mathrm{i} k_{0}\left|\mathbf{r}-\mathbf{r}^{\prime}\right|\right) / 4 \pi\left|\mathbf{r}-\mathbf{r}^{\prime}\right|$ is the three dimensional scalar free space Green's function for the Helmholtz equation.

Hence, the general solution of (1) for the case of the electric field $\mathbf{E}(\mathbf{r})$ in integral form can be summarized as

$$
\begin{aligned}
\mathbf{E}(\mathbf{r}) & =\mathbf{E}_{i}(\mathbf{r})+\int_{V} \overline{\mathbf{G}}\left(\mathbf{r}, \mathbf{r}^{\prime}\right) \\
& \left\{k_{0}^{2}\left(n^{2}-1\right) \mathbf{E}\left(\mathbf{r}^{\prime}\right)+\nabla \times\left[\left(1-\mu^{-1}\right) \nabla^{\prime} \times \mathbf{E}\left(\mathbf{r}^{\prime}\right)\right]\right\} \mathrm{d}^{3} r^{\prime},
\end{aligned}
$$

where $\gamma(\mathbf{r})=k_{0}^{2}\left(n^{2}-1\right)$ is the polarizability of the medium assumed dispersion-free and the integral extends over the volume of the scatterer and $\mathbf{E}_{i}(\mathbf{r})$ is the incident electric filed. In case of dielectric particles (i.e. non-magnetic particles $\mu \approx 1$ ), the integral equation reduces to the form

$$
\mathbf{E}(\mathbf{r})=\mathbf{E}_{i}(\mathbf{r})+\int_{V} \overline{\mathbf{G}}\left(\mathbf{r}, \mathbf{r}^{\prime}\right) k_{0}^{2}\left(n^{2}-1\right) \mathbf{E}\left(\mathbf{r}^{\prime}\right) \mathrm{d}^{3} r^{\prime} .
$$

The incident plane wave $\mathbf{E}_{i}(\mathbf{r})$ can be defined in dyadic notation as:

$$
\mathbf{E}_{i}(\mathbf{r})=\overline{\mathbf{J}}_{i} \exp \left(\mathrm{i} \mathbf{k}_{i} \cdot \mathbf{r}\right),
$$

where $\overline{\mathbf{J}}_{i}=\overline{\mathbf{I}}-\hat{\mathbf{k}}_{i} \hat{\mathbf{k}}_{i}$ is the projection dyadic relative to the incident field direction, $\hat{\mathbf{k}}_{i}$ is a unit vector along the incident wavevector $\mathbf{k}_{i}$.

Using the asymptotic behaviour of $\overline{\mathbf{G}}\left(\mathbf{r}, \mathbf{r}^{\prime}\right)$, the electric field $\mathbf{E}(\mathbf{r})$ in the far field is given as

$$
\mathbf{E}(\mathbf{r}) \approx \overline{\mathbf{J}}_{i} \exp \left(\mathrm{i} \mathbf{k}_{i} \cdot \mathbf{r}\right)+\frac{\exp \left(\mathrm{i} k_{0} r\right)}{r} \overline{\mathbf{F}}\left(\mathbf{k}_{s}, \mathbf{k}_{i}\right)+O\left(\frac{1}{r^{2}}\right),
$$

where $\mathbf{k}_{s}$ is the scattered wave vector, and $\overline{\mathbf{F}}\left(\mathbf{k}_{s}, \mathbf{k}_{i}\right)$ is the dyadic scattering amplitude defined as

$$
\overline{\mathbf{F}}\left(\mathbf{k}_{s}, \mathbf{k}_{i}\right)=\overline{\mathbf{J}}_{s} \int_{V} \exp \left(-\mathrm{i} \mathbf{k}_{s} \cdot \mathbf{r}\right) \gamma(\mathbf{r}) \mathbf{E}(\mathbf{r}) \mathrm{d}^{3} r .
$$

Importantly, the dyadic scattering amplitude is dependent only on the electric field within the volume of the scatterer. The 
field equation (6) is an integral equation with a singular kernel. In order to find an analytical solution, the FIM method is proposed in [29] to deal with the singularity.

Multiplying (6) by $\exp \left(-\mathrm{i} \mathbf{k}_{1} \cdot \mathbf{r}\right) \gamma(\mathbf{r})$ and integrating over the volume of the scatterer we obtain a new equation that we omit for brevity. This equation does not have a unique solution $\mathbf{E}(\mathbf{r})$. This is because the equation contains only the field inside the scatterer so that fields differing in behaviours outside the scatterer are all solutions [19]. However, since the scattering amplitude (9) is computed as an integral over only the volume of the scatterer, any solution $\mathbf{E}(\mathbf{r})$ of the new equation substituted in (9) will produce a unique scattering amplitude. Assuming a square-integrable solution of the new equation, it can be expressed as a Fourier transform

$$
\mathbf{E}(\mathbf{r})=\int \overline{\mathbf{C}}\left(\mathbf{k}_{2}\right) \exp \left(-\mathrm{i} \mathbf{k}_{2} \cdot \mathbf{r}\right) \mathrm{d}^{3} k_{2} .
$$

Substituting this solution in the new equation we obtain

$$
\int \overline{\mathbf{K}}\left(\mathbf{k}_{1}, \mathbf{k}_{2}\right) \overline{\mathbf{C}}\left(\mathbf{k}_{2}\right) \mathrm{d}^{3} k_{2}=\overline{\mathbf{J}}_{i} U\left(\mathbf{k}_{1}, \mathbf{k}_{i}\right)
$$

where

$$
U\left(\mathbf{k}_{1}, \mathbf{k}_{i}\right)=\int \gamma(\mathbf{r}) \exp \left[-\mathrm{i}\left(\mathbf{k}_{1}-\mathbf{k}_{i}\right) \cdot \mathbf{r}\right] \mathrm{d}^{3} r
$$

$U\left(\mathbf{k}_{1}, \mathbf{k}_{i}\right)$ is the first Born term which in effect is the spatial Fourier transform of the polarizability $\gamma(\mathbf{r})$ of the scatterer. The $\overline{\mathbf{K}}$ term in (11) is the kernel of the integral equation and found to be remarkably stable in [19]. It can be expressed as

$$
\begin{array}{r}
\overline{\mathbf{K}}\left(\mathbf{k}_{1}, \mathbf{k}_{2}\right)=\overline{\mathbf{I}} U\left(\mathbf{k}_{1}, \mathbf{k}_{2}\right)-\iint \gamma(\mathbf{r}) \gamma\left(\mathbf{r}^{\prime}\right) \\
\exp \left(-\mathrm{i} \mathbf{k}_{1} \cdot \mathbf{r}\right) \overline{\mathbf{G}}\left(\mathbf{r}, \mathbf{r}^{\prime}\right) \mathrm{e}^{\mathrm{i} \mathbf{k}_{2} \cdot \mathbf{r}^{\prime}} \mathrm{d}^{3} r \mathrm{~d}^{3} r^{\prime}
\end{array}
$$

or alternatively

$$
\overline{\mathbf{K}}\left(\mathbf{k}_{1}, \mathbf{k}_{2}\right)=\overline{\mathbf{I}} W\left(\mathbf{k}_{1}, \mathbf{k}_{2}\right)-\overline{\mathbf{Z}}\left(\mathbf{k}_{1}, \mathbf{k}_{2}\right),
$$

where $W\left(\mathbf{k}_{1}, \mathbf{k}_{2}\right)$, in the case of homogeneous scatterers, is the integral of a pointwise multiplication of the first Born term $U\left(\mathbf{k}_{1}, \mathbf{k}_{2}\right)$ with the dielectric constant [30]:

$$
W\left(\mathbf{k}_{1}, \mathbf{k}_{2}\right)=\int \exp \left[-\mathrm{i}\left(\mathbf{k}_{1}-\mathbf{k}_{2}\right) \cdot \mathbf{r}\right] \gamma(\mathbf{r}) \varepsilon(\mathbf{r}) \mathrm{d}^{3} r,
$$

and $\overline{\mathbf{Z}}\left(\mathbf{k}_{1}, \mathbf{k}_{2}\right)$ is the the second Born term:

$$
\begin{aligned}
\overline{\mathbf{Z}}\left(\mathbf{k}_{1}, \mathbf{k}_{2}\right)= & \frac{1}{8 \pi^{3} k_{0}^{2}} \lim _{\xi \rightarrow 0} \int \frac{p^{2} \mathrm{~d}^{3} p}{p^{2}-k_{0}^{2}-\mathrm{i} \xi} \\
& {[\overline{\mathbf{I}}-\hat{\mathbf{p}} \hat{\mathbf{p}}] U\left(\mathbf{k}_{1}, \mathbf{p}\right) U\left(\mathbf{p}, \mathbf{k}_{2}\right) . }
\end{aligned}
$$

In (16) the vector $\mathbf{p}$ is a pivot vector and the parameter $\xi$ has been introduced to mitigate the singularity of the function to integrate. Substituting the assumed solution (10) in the expression of the scattering amplitude (9) we obtain the dyadic scattering amplitude

$$
\overline{\mathbf{F}}\left(\mathbf{k}_{s}, \mathbf{k}_{i}\right)=\frac{1}{4 \pi} \overline{\mathbf{J}}_{s} \int U\left(\mathbf{k}_{s}, \mathbf{k}_{2}\right) \overline{\mathbf{C}}\left(\mathbf{k}_{2}\right) \mathrm{d}^{3} k_{2} .
$$

Equations (11) and (17) are the coupled Fredholm integral equations for the problem. Equation (11) is used to compute $\overline{\mathbf{C}}\left(\mathbf{k}_{2}\right)$ and (17) is then used to computed the scattering amplitude.
They have the important property that now the kernel $\overline{\mathbf{K}}$ in (11) is non-singular and (13) can be computed with the numerical quadrature method. Hence, the integral equation (11) can be reduced to a matrix equation which is easily solved using any of the established factorization methods.

The evaluation of the first Born term (12) is straight forward. Instead, the second Born term (16) is more complex to evaluate. In [19] and [20] the Z-term (16) is calculated by expanding the two first Born terms into series and then carrying out the contour integration of the infinite radial integral as in [31] and [32]. The FIM is an accurate method to evaluate the scattering of an electromagnetic wave by homogeneous dielectric spheroids or ellipsoids. However, especially in the case of large particles the evaluation of the second Born term by expansion of the first Born term may require too large machine storage [19]. Moreover, the evaluation of the scattering amplitude for inhomogeneous particles has not been carried out with this method and inaccuracies may arise due to the necessity of using mixing theories to estimate the effective dielectric constant.

\section{A. The Discrete Method}

The DM was pioneered in [24] and [33] with the aim to tackle the computation of the scattering amplitude for the inhomogeneous particle by discretizing the inhomogeneous scatterer into homogeneous cells. The process of discretization enables the computation of the scattering amplitude for inhomogeneous particles without using mixing theories. Moreover, with the discretization the computation of the total field is carried out calculating the field only for one homogeneous cell at the origin for each dielectric constant present within the particle. The contribution to the total field of all the other cells is simply obtained with the Fourier shift theorem.

Let us consider a finite domain of three-dimensional regular lattice field discretized into cubic cells or grid points as shown in Fig. 1 where the cubic cell has been replaced by a spherical cell. This approximation will be very relevant in the calculation of the second Born term and it has been used in DDA method [18].

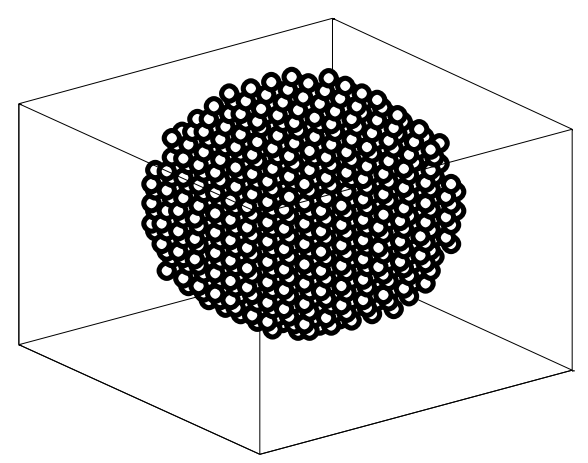

Fig. 1. The discretized regular lattice field for a spherical particle.

The discretization applies correspondingly in the Fourier domain and it is shown in Fig. 2 


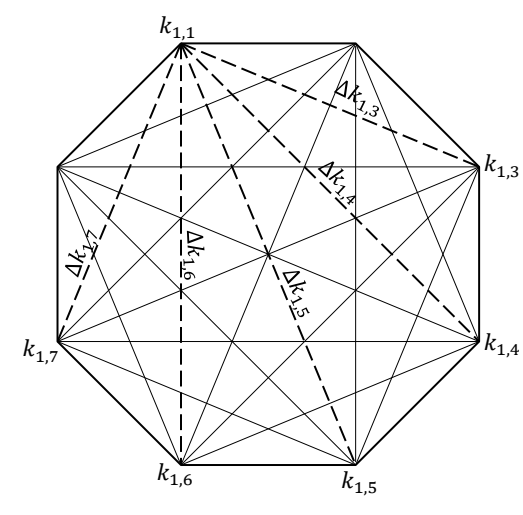

Fig. 2. Cross-sectional view of the discretized regular lattice field in the Fourier domain for 8 internal wavevector where $\Delta \mathbf{k}_{l, m}$ is the distance between $\mathbf{k}_{l}$ and $\mathbf{k}_{m}$ for $l=1$ and $m=1, \ldots 8$.

\section{B. Evaluation of the first Born term}

For the evaluation of first Born term in (12) the discretization of the lattice field simplifies the computation of $U$ as a summation over the cells according to their dielectric properties $\gamma\left(\mathbf{r}_{j}\right)$ (weighted contents). The first Born term with respect to the internal wavevectors $\mathbf{k}_{l}$ and $\mathbf{k}_{m}$

$$
U\left(\mathbf{k}_{l}, \mathbf{k}_{m}\right)=\sum_{j} U_{j}\left(\mathbf{k}_{l}, \mathbf{k}_{m}\right),
$$

where the sum is over all the cells and

$$
\begin{aligned}
U_{j}\left(\mathbf{k}_{l}, \mathbf{k}_{m}\right) & =\exp \left[-\mathrm{i}\left(\mathbf{k}_{l}-\mathbf{k}_{m}\right) \cdot \mathbf{r}_{j}\right] \gamma\left(\mathbf{r}_{j}\right) \\
& \int_{\mathbf{r} \subset V_{j}} \exp \left[-\mathrm{i}\left(\mathbf{k}_{l}-\mathbf{k}_{m}\right) \cdot\left(\mathbf{r}-\mathbf{r}_{j}\right)\right] \mathrm{d}^{3} r \\
& =\exp \left[-\mathrm{i}\left(\mathbf{k}_{l}-\mathbf{k}_{m}\right) \cdot \mathbf{r}_{j}\right] \gamma\left(\mathbf{r}_{j}\right) U_{0}\left(\mathbf{k}_{l}, \mathbf{k}_{m}\right) .
\end{aligned}
$$

where $V_{j}$ is the volume of the $j^{t h}$ cell, $\mathbf{r}_{j}$ is the centre of the $j^{\text {th }}$ cell, and $U_{0}$ is

$$
U_{0}\left(\mathbf{k}_{l}, \mathbf{k}_{m}\right)=\int_{\mathbf{r} \subset V_{j}} \exp \left[-\mathrm{i}\left(\mathbf{k}_{l}-\mathbf{k}_{m}\right) \cdot\left(\mathbf{r}-\mathbf{r}_{j}\right)\right] \mathrm{d}^{3} r .
$$

The $U_{0}\left(\mathbf{k}_{l}, \mathbf{k}_{m}\right)$ in (20) is the Fourier transform of the weighted cell, and it is a spatially invariant function of $\mathbf{k}_{l}-\mathbf{k}_{m}$. For small size parameter where $\left|\mathbf{k}_{l}\right|=\left|\mathbf{k}_{m}\right| \approx\left|\mathbf{k}_{0}\right|$, $U_{0}\left(\mathbf{k}_{l}, \mathbf{k}_{m}\right)$ is well approximated by the cell volume $\Delta v$ and (19) becomes

$$
U_{j}\left(\mathbf{k}_{l}, \mathbf{k}_{m}\right)=\exp \left(-\mathrm{i} \Delta \mathbf{k}_{l, m} \cdot \mathbf{r}_{j}\right) \gamma\left(\mathbf{r}_{j}\right) \Delta v,
$$

where $\Delta \mathbf{k}_{l, m}=\mathbf{k}_{l}-\mathbf{k}_{m}$ is the distance between the internal wavevectors and $\exp \left(-\mathrm{i} \Delta \mathbf{k}_{l, m} \cdot \mathbf{r}_{j}\right)$ is the phase shift for each grid point in the 3 -dimensional regular lattice field. The calculation of the term (21) for any other cell in the lattice field can be easily done reusing the numerical values of $\Delta v$ and $\Delta \mathbf{k}_{l, m}$ computed for the first cell.

The $W\left(\mathbf{k}_{l}, \mathbf{k}_{m}\right)$ term (15) follows the same procedure of the U-term. In fact, $W$ can be obtained with a simple pointwise multiplication of the dielectric constant $\varepsilon(\mathbf{r})$ at the grid points within the scatterer by the first Born term in (18):

$$
W\left(\mathbf{k}_{l}, \mathbf{k}_{m}\right)=\sum_{j} \exp \left(-\mathrm{i} \Delta \mathbf{k}_{l, m} \cdot \mathbf{r}_{j}\right) \gamma\left(\mathbf{r}_{j}\right) \varepsilon\left(\mathbf{r}_{j}\right) \Delta v,
$$

or, alternatively as

$$
W\left(\mathbf{k}_{l}, \mathbf{k}_{m}\right)=\sum_{j} U_{j}\left(\mathbf{k}_{l}, \mathbf{k}_{m}\right) \varepsilon\left(\mathbf{r}_{j}\right)
$$

\section{Evaluation of the second Born term}

Regarding the second Born term (16), we apply the same assumption of uniform discretization of the regular lattice field as for the U-term. The discretization applies to the two Uterms that appear under the integral:

$$
\begin{array}{r}
\bar{Z}\left(\mathbf{k}_{l}, \mathbf{k}_{m}\right)=\frac{1}{8 \pi^{3} k_{0}^{2}} \lim _{\xi \rightarrow 0} \sum_{j, i} \int_{\Omega} \int_{0}^{\infty} \frac{p^{4} \mathrm{~d} \Omega \mathrm{d} p}{p^{2}-k_{0}^{2}-\mathrm{i} \xi} \\
{[\overline{\mathbf{I}}-\hat{\mathbf{p}} \hat{\mathbf{p}}] U_{j}\left(\mathbf{k}_{l}, \mathbf{p}\right) U_{i}\left(\mathbf{p}, \mathbf{k}_{m}\right) .}
\end{array}
$$

where the U-terms are as in (19)

$$
U_{j}\left(\mathbf{k}_{l}, \mathbf{p}\right)=\gamma\left(\mathbf{r}_{j}\right) \exp \left(-\mathrm{i}\left(\mathbf{k}_{l}-\mathbf{p}\right) \cdot \mathbf{r}_{j}\right) U_{0}\left(\mathbf{k}_{l}, \mathbf{p}\right),
$$

and

$$
U_{i}\left(\mathbf{p}, \mathbf{k}_{m}\right)=\gamma\left(\mathbf{r}_{i}\right) \exp \left(-\mathrm{i}\left(\mathbf{p}-\mathbf{k}_{m}\right) \cdot \mathbf{r}_{i}\right) U_{0}\left(\mathbf{p}, \mathbf{k}_{m}\right) .
$$

Substituting (25) and (26) into (24) leads to expressing the Z-term as

$$
\begin{aligned}
\bar{Z}\left(\mathbf{k}_{l}, \mathbf{k}_{m}\right) & =\frac{1}{8 \pi^{3} k_{0}^{2}} \lim _{\xi \rightarrow 0} \sum_{j, i} \gamma\left(\mathbf{r}_{j}\right) \gamma\left(\mathbf{r}_{i}\right) \\
& \int_{\Omega} \int_{0}^{\infty} \mathrm{e}^{\left[-\mathrm{i}\left(\mathbf{k}_{l}-\mathbf{p}\right) \cdot \mathbf{r}_{j}\right]} \mathrm{e}^{\left[-\mathrm{i}\left(\mathbf{p}-\mathbf{k}_{m}\right) \cdot \mathbf{r}_{i}\right]} \\
& U_{0}\left(\mathbf{k}_{l}, \mathbf{p}\right) U_{0}\left(\mathbf{p}, \mathbf{k}_{m}\right) \frac{p^{4} \mathrm{~d} \Omega \mathrm{d} p}{p^{2}-k_{0}^{2}-\mathrm{i} \xi}[\overline{\mathbf{I}}-\hat{\mathbf{p}} \hat{\mathbf{p}}],
\end{aligned}
$$

where $\overline{\mathbf{I}}-\hat{\mathbf{p}} \hat{\mathbf{p}}$ can be expressed as

$$
\overline{\mathbf{I}}-\hat{\mathbf{p}} \hat{\mathbf{p}}=\left(\begin{array}{ccc}
1-y^{2} \cos ^{2} \phi & -y^{2} \cos \phi \sin \phi & -x y \cos \phi \\
-y^{2} \cos \phi \sin \phi & 1-y^{2} \sin ^{2} \phi & -x y \sin \phi \\
-x y \cos \phi & -x y \sin \phi & y^{2}
\end{array}\right),
$$

and we have used the following: $\cos \theta=x, \sin \theta=y$. Rearranging the terms in (27), the Z-term is expressed as

$$
\begin{aligned}
\overline{\mathbf{Z}}\left(\mathbf{k}_{l}, \mathbf{k}_{m}\right) & =\frac{1}{8 \pi^{3} k_{0}^{2}} \lim _{\xi \rightarrow 0} \sum_{j, i} \gamma\left(\mathbf{r}_{j}\right) \gamma\left(\mathbf{r}_{i}\right) \exp \left(-\mathrm{i} \mathbf{k}_{l} \cdot \mathbf{r}_{j}\right) \\
& \exp \left(\mathrm{i} \mathbf{k}_{m} \cdot \mathbf{r}_{i}\right) \int_{\Omega} \int_{0}^{\infty} \exp \left[\mathrm{i}\left(\hat{\mathbf{p}} \cdot\left(\mathbf{r}_{j}-\mathbf{r}_{i}\right) p\right]\right. \\
& U_{0}\left(\mathbf{k}_{l}, \mathbf{p}\right) U_{0}\left(\mathbf{p}, \mathbf{k}_{m}\right) \frac{p^{4} \mathrm{~d} \Omega \mathrm{d} p}{p^{2}-k_{0}^{2}-\mathrm{i} \xi}[\overline{\mathbf{I}}-\hat{\mathbf{p}} \hat{\mathbf{p}}],
\end{aligned}
$$

where $\gamma\left(\mathbf{r}_{j}\right)$ and $\gamma\left(\mathbf{r}_{i}\right)$ are the polarizabilities given as

$$
\gamma\left(\mathbf{r}_{i}\right)=k_{0}^{2}\left(\varepsilon\left(\mathbf{r}_{i}\right)-1\right)
$$

with respect to distinct cells in the regular 3-dimensional lattice field. In order to calculate the Z-term, we need to evaluate the integral over $p$ by contour integration:

$$
\int_{\Omega} \int_{0}^{\infty} U_{0}\left(\mathbf{k}_{l}, \mathbf{p}\right) U_{0}\left(\mathbf{p}, \mathbf{k}_{m}\right) \exp (\mathrm{i} \beta p) \frac{p^{4} \mathrm{~d} \Omega \mathrm{d} p}{p^{2}-k_{0}^{2}-\mathrm{i} \xi},
$$

where $\beta=\hat{\mathbf{p}} \cdot\left(\mathbf{r}_{i}-\mathbf{r}_{j}\right)$ is the relative phase of the cells measured in the direction of the pivot vector. The largest contribution to this integral occurs close to the pole $p=k_{0}$ 
where $U_{0}$ is well approximated by the elementary volume. However, a problem arises along the imaginary axis where the integrand exhibits singularities. For a cubic cell, $U_{0}$ takes the form of a three dimensional sinc function which is difficult to integrate analytically since it does not converge, as also discussed in [34].

Now, we consider a more radical approach in which it is argued that the contribution of the U-terms should not depend critically on the shape of the cell, but mainly on its volume. Accordingly, we consider instead spherical cells of the same volume as the cubes. We compute $U_{s}$ which is the term $U_{0}$ for a sphere of equal volume to the one of basic cubic cell verifying that this term does not produce singularities at large imaginary $p$. Hence, $U_{0}$ for a spherical cell of radius $\rho$ becomes

$$
U_{0} \approx U_{s}=4 \pi \rho^{3} \frac{J_{\frac{3}{2}}(p \rho)}{(p \rho)^{\frac{3}{2}}},
$$

where $J_{\frac{3}{2}}(p \rho)$ is the half-order Bessel function. $\rho$ is the equivalent volume radius of the cell which can be related to the dimensions $a, b, c$ of the cubic cell as

$$
a b c=\frac{4 \pi \rho^{3}}{3}, \quad \text { if } a=b=c, \quad a^{3}=\frac{4 \pi \rho^{3}}{3},
$$

so that the equivalent volume radius $\rho$ is expressed as

$$
\rho=a\left(\sqrt[3]{\frac{3}{4 \pi}}\right)
$$

When the argument $p \rho$ in (32) is very small (i.e. zero of the Fourier transform of the content of the sphere), $U_{s} \approx 4 \pi \rho^{3} \frac{1}{3}$ which is the spherical cell volume.

In the calculation of (32) we have neglected the offset contribution of $\mathbf{k}_{l}$ and $\mathbf{k}_{m}$ [31]. This can be justified by considering an expansion in infinite series of higher order cylinder functions of (20) where it can be assumed that $p \rho$ is so small for the fundamental cell that the higher terms can be neglected.

Substituting (32) into (31) gives the integral $I_{k_{0}}$

$$
I_{k_{0}}=\lim _{\xi \longrightarrow 0+} \frac{1}{\rho^{3}} \int_{0}^{\infty} \frac{J_{\nu}^{2}(p \rho)}{p^{3}} \frac{p^{4} \exp (\mathrm{i} \beta p)}{p^{2}-k_{0}^{2}-\mathrm{i} \xi} d p
$$

which is an integral of products of Bessel functions of halforder $\nu=\frac{3}{2}$. However, it is not possible to obtain a closed form analytical solution of this integral. Let us see in details the reasons. The integrand function shows a singularity for $p=k_{0}$ close to the real axis. Integrals of this type were considered early in the history of the analysis of Bessel functions, and solved using the Hankel integral method [31, p.429]. In many cases, such integrals of products of Bessel functions have a closed form solution which can be obtained evaluating Bessel and Hankel functions at the pole. However, this approach [31], [32] cannot be applied because of the power of $p$ in the integrand being odd. In this case, it is not possible to use symmetry arguments to extend the integral to the negative axis.

The alternative approach that we propose is based on a truncation and still involves contour integration method. If the cell sizes are small enough with respect to the wavelength, we can expand the Bessel functions and truncate the expansion at the first degree [31]. Let us consider the following integral

$$
I_{1}=\lim _{\xi \longrightarrow 0+} \frac{1}{\rho^{3}} \int_{0}^{\infty} J_{\nu}^{2}\left(\rho k_{0} p^{\prime}\right) \frac{p^{\prime} \exp \left(\mathrm{i} \beta k_{0} p^{\prime}\right)}{p^{2}-1-\mathrm{i} \xi} d p^{\prime},
$$

where

$$
p^{\prime}=\frac{p}{k_{0}} \text {. }
$$

Hence, comparing (35) and (36), we can write $I_{k_{0}}$ as

$$
I_{k_{0}}=\frac{1}{k_{0}^{2}} I_{1} \text {. }
$$

Then, substituting the expression of $I_{k_{0}}$ as in (38) into (29) leads to expressing the Z-term as

$$
\begin{aligned}
Z\left(\mathbf{k}_{l}, \mathbf{k}_{m}\right)= & \frac{1}{8 \pi^{3} k_{0}^{2}} \frac{4 \pi}{N_{m}} \frac{16 \pi^{2} \rho^{6}}{\rho^{3}} \sum_{j, i} \gamma\left(\mathbf{r}_{j}\right) \gamma\left(\mathbf{r}_{i}\right) \\
& \int_{\Omega} \exp \left(-\mathrm{i} \mathbf{k}_{l} \cdot \mathbf{r}_{j}\right) \exp \left(\mathrm{i} \mathbf{k}_{m} \cdot \mathbf{r}_{i}\right)[\overline{\mathbf{I}}-\hat{\mathbf{p}} \hat{\mathbf{p}}] \\
& \int_{0}^{\infty} J_{\nu}^{2}\left(\rho k_{0} p^{\prime}\right) \frac{p^{\prime} \exp \left(\mathrm{i} \beta k_{0} p^{\prime}\right)}{p^{\prime 2}-1} d \Omega d p^{\prime},
\end{aligned}
$$

where $N_{m}$ is the number of angular integrals for all the cells. Equation (39) contains the normalised form $I_{1}$ of the integral required in evaluating the Z-term.

An approach to solve $I_{1}$ would be to integrate along a quandrantal contour which goes from zero to real infinity, then anticlockwise around a quarter circle and then returning along the imaginary axis as shown in Fig. 3.

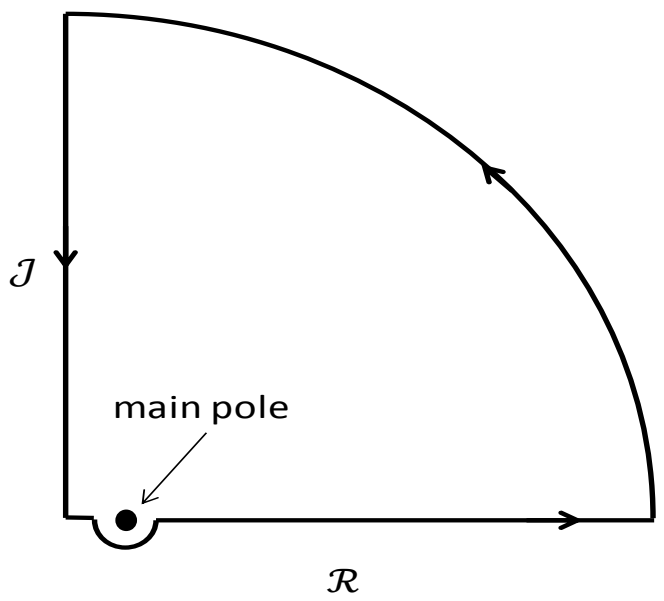

Fig. 3. Semi circle split into two equal half with the top half enclosing the poles at near real axis.

The attractiveness of this scheme would be that the integral along the imaginary axis has no poles and decreases monotonically. However, on the imaginary axis the integral does not converged.

A more workable solution was found by using a conditioning weighting function in the radial integral (35) which takes the form,

$$
\int_{0}^{\infty} J_{\nu}^{2}\left(k_{0} \rho p^{\prime}\right) w\left(p^{\prime}\right) \frac{p^{\prime} \exp \left(\mathrm{i} \beta k_{0} p^{\prime}\right)}{p^{2}-1} d p^{\prime} .
$$

This function does not alter the result of the integral significantly in the integration region and it contributes to the 
elimination of the singularities as explained below. The conditioning weighting function $w\left(p^{\prime}\right)$ can be expressed as

$$
w\left(p^{\prime}\right)=\frac{1}{1+\exp \left(-\left(\alpha p^{\prime}\right)^{2}\right)},
$$

where $p^{\prime}$ in (41) is complex and it is defined as

$$
\begin{aligned}
p^{\prime} & =\frac{1}{\alpha} \sqrt{(2 n-1) \pi i}= \\
& =\frac{1}{\alpha} \sqrt{(2 n-1) \pi} \frac{(1+i)}{\sqrt{2}} \quad n=1,2,3,4, \cdots, N .
\end{aligned}
$$

. The function $w$ on the real axis, where $p^{\prime}=x$ takes this form

$$
w(x)=\frac{1}{1+\exp \left(-\alpha^{2} x^{2}\right)} .
$$

It behaves well near the origin $w(x) \approx \frac{1}{2}$, and rapidly increases to 1 as shown in Fig. 4. Therefore, the weighting function does not contribute significantly to the original integral and (40) approximates well the desired integral in (39) for a large range of values of $\alpha$. Moreover, since the integral is small anyway near the origin, the result is accurate even for very modest values of $\alpha$.

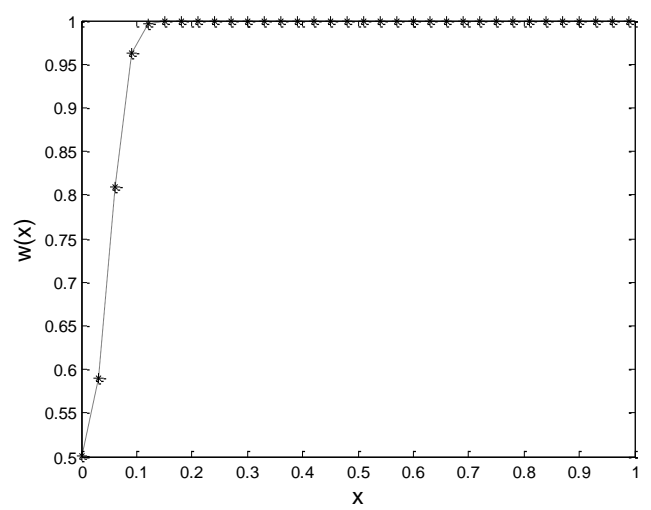

Fig. 4. The weighting factor $w$ as a function of $\Re\left(p^{\prime}\right)$ for $\alpha=4.0$.

Along the imaginary axis, where $p^{\prime}=i y, w$ takes the form

$$
w(y)=\frac{1}{1+\exp \left(\alpha^{2} y^{2}\right)} .
$$

For small values of $y, w \approx \frac{1}{2}$ and it decreases very rapidly to zero as shown in Fig. 5. Even though the integral in (40) might increase exponentially along the imaginary axis, $w$ decreases much faster. In fact, along the imaginary axis the weighting function very rapidly tends to zero, and apart from a similar very small contribution near the origin, the weighted integral on the imaginary axis becomes negligible.

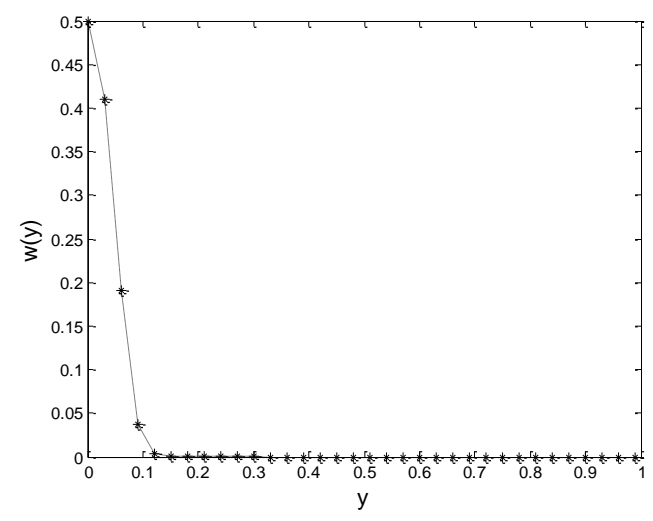

Fig. 5. The weighting factor $w$ as a function of $\Im\left(p^{\prime}\right)$ for $\alpha=4.0$.

As a result, therefore, the desired integral can be expressed accurately as a sum of residues. However, the weighting function itself introduces new singularities, in fact an infinity of them along the line $x=y$ as shown in Fig. 6. Experimental results show that the number of weighting function poles, $n_{p^{\prime}} \geq 100$ are sufficient to perform the integration.

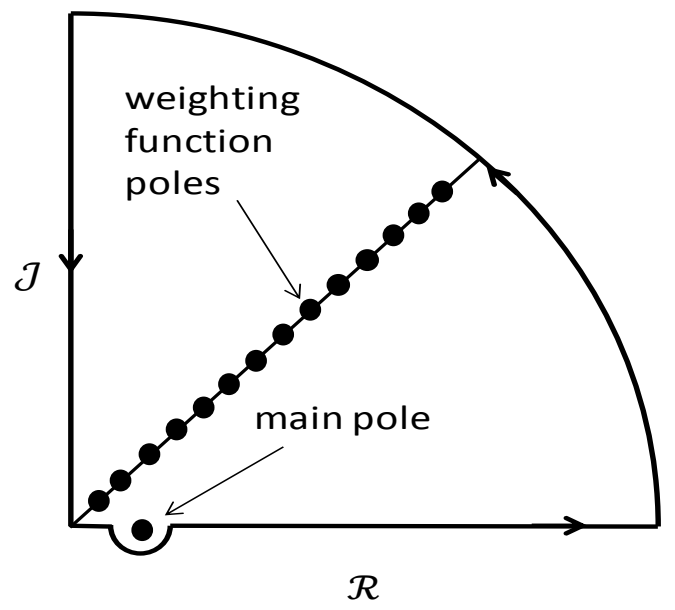

Fig. 6. Semi circle split into two equal half with the top half enclosing the poles at near real axis and the weighting function poles at $x=y$ for the evaluation of the second Born term.

Although it is necessary to sum typically one hundred residues to obtain convergence, the regularity of the spacing of the poles makes the sum typically scalable in relation to the cell size, and by considering the sum of the residues as a function in its own right, it has been found that for a wide range of parameters $\rho$ and $\beta$, the residue sum can be approximated piecewise as a function of $\rho$ and $\beta$. An exception to this finding, however, occurs when the phase separation parameter $\beta$ is small. In this regime it appears that the summation of the residues fails to converge, and the only viable alternative appears to directly model the function. Numerical experimentation established that in this regime the function has excellent scaling properties with respect to cell size (when the cell is small) so that a universal function of one parameter can effectively be established. Because the function has a real symmetric peak and an antisymmetric imaginary part that also decays rapidly, the primary model for the function 
was a Gaussian for the real part and the derivative of a Gaussian for the imaginary part. In practice this was refined by fitting quartic functions to the argument of the exponential factors.

Finally, the Z-term including the conditioning weighting function is summarised as

$$
\begin{aligned}
Z\left(\mathbf{k}_{l}, \mathbf{k}_{m}\right) & =\frac{1}{2 \pi^{2} k_{0}^{2} N_{m}} 12 \pi \Delta v \sum_{j, i} \gamma\left(\mathbf{r}_{j}\right) \gamma\left(\mathbf{r}_{i}\right) \\
& \int_{\Omega} \exp \left(-\mathrm{i} \mathbf{k}_{l} \cdot \mathbf{r}_{j}\right) \exp \left(\mathrm{i} \mathbf{k}_{m} \cdot \mathbf{r}_{i}\right)[\overline{\mathbf{I}}-\hat{\mathbf{p}} \hat{\mathbf{p}}] \\
& \int_{0}^{\infty} J_{\nu}^{2}\left(\rho k_{0} p^{\prime}\right) w\left(p^{\prime}\right) \frac{p^{\prime} \exp \left(\mathrm{i} \beta k_{0} p^{\prime}\right)}{p^{\prime 2}-1} d \Omega d p^{\prime},
\end{aligned}
$$

where $\Delta v=\frac{4 \pi \rho^{3}}{3}$.

\section{Evaluation of the scattering amplitude}

To evaluate the scattering amplitude, firstly the non-singular kernel $\overline{\mathbf{K}}$ is computed as in (14) which for our Discrete Method takes the form:

$$
\overline{\mathbf{K}}\left(\mathbf{k}_{l}, \mathbf{k}_{m}\right)=\overline{\mathbf{I}} W\left(\mathbf{k}_{l}, \mathbf{k}_{m}\right)-\overline{\mathbf{Z}}\left(\mathbf{k}_{l}, \mathbf{k}_{m}\right) .
$$

Now, it is possible to evaluate the unknown vector $\overline{\mathbf{C}}_{m}$ in (11). Using the Discrete Method, equation (11) can be simplified and it reduces to a linear block matrix equation with dyadic elements. The equation takes the form

$$
\begin{aligned}
\sum_{l, m} \overline{\mathbf{K}}\left(\mathbf{k}_{l}, \mathbf{k}_{m}\right)\left(w_{m} \overline{\mathbf{C}}_{m}\right) & =\overline{\mathbf{J}}_{i} U\left(\mathbf{k}_{l}, \mathbf{k}_{i}\right), \\
\sum_{l, m} \overline{\mathbf{K}}\left(\mathbf{k}_{l}, \mathbf{k}_{m}\right) \overline{\mathbf{Y}}_{m} & =\overline{\mathbf{J}}_{l}
\end{aligned}
$$

where $\mathbf{k}_{l}$ and $\mathbf{k}_{m}$ are the internal wavevectors, $\mathbf{k}_{i}$ is the incident wave vector, $\overline{\mathbf{J}}_{i}=\left[\overline{\mathbf{I}}-\hat{\mathbf{k}}_{i} \hat{\mathbf{k}}_{i}\right]$ is the projection vector along the incident field direction, $U\left(\mathbf{k}_{l}, \mathbf{k}_{i}\right)$ is a first Born term with respect to the internal wavevectors and incident wavevector, $w_{m}=\frac{4 \pi}{N_{m}}$ is the numerical integration weighting factor with $N_{m}$ the number of integral over $p$ and $\overline{\mathbf{Y}}_{m}=w_{m} \overline{\mathbf{C}}_{m}$.

After some mathematical manipulation, we can write (9) as a summation over all the cells and multiply by scattered direction projection vector $\overline{\mathbf{J}}_{s}$ to obtain the scattering amplitude $\overline{\mathbf{F}}$ as

$$
\overline{\mathbf{F}}\left(\mathbf{k}_{s}, \mathbf{k}_{i}\right)=\overline{\mathbf{J}}_{s} \sum_{m} U_{j}\left(\mathbf{k}_{s}, \mathbf{k}_{m}\right) \overline{\mathbf{Y}}_{m}
$$

where $\mathbf{k}_{s}$ is the scattered wavevector, and $\overline{\mathbf{J}}_{s}=\left[\overline{\mathbf{I}}-\hat{\mathbf{k}}_{s} \hat{\mathbf{k}}_{s}\right]$ is the dyadic projection vector in the scattered field direction.

Equations (47) and (48) are the coupled Fredholm integral equations in discrete form. Together they determine the scattering amplitude function which can be obtained solving only one integral while all the other computations have been reduced to simple matrix manipulations. This method is more attractive than the previous in [19] since it is quicker to implement and much faster to run despite the solution is approximated through discretization unlike in [19]. The other scattering properties can easily be obtained once the dyadic scattering amplitude function is evaluated.

\section{VAlidation of the Dicrete Method}

The validation of the Dicrete Method is carried out for spherical particles firstly comparing the first Born term with the one evaluated with the FIM by [19] and then comparing the scattering cross section evaluated with the DM and the Mie theory.

The homogeneous spherical particles are modelled as ellipsoids

$$
\frac{x^{2}}{a^{2}}+\frac{y^{2}}{b^{2}}+\frac{z^{2}}{c^{2}} \leq 1
$$

since ellipsoids are of particular interest in weather radar applications where precipitation tends to be represented with such a shape [35], [36]. Each ellipsoidal particle is filled with spherical cells according to $\gamma\left(\mathbf{r}_{i}\right)$ (30) which characterises the medium of each cell. For homogeneous particles $\gamma\left(\mathbf{r}_{i}\right)$ is the same for all the cell.

\section{A. Validation of the first Born term}

The initial validation was carried out by comparing the first Born term computed using our method (18) and the method (12) by Holt et.al [19]. Numerical results with different number of pivots are shown in Fig. 7 - 8 and they show a good agreement independently on the number of pivot.

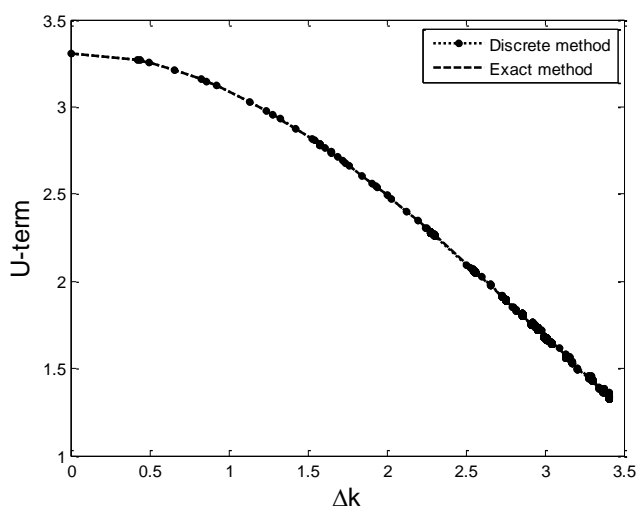

Fig. 7. Comparison of first Born term using the Holt and the Discrete (with 24 internal wavectors) methods.

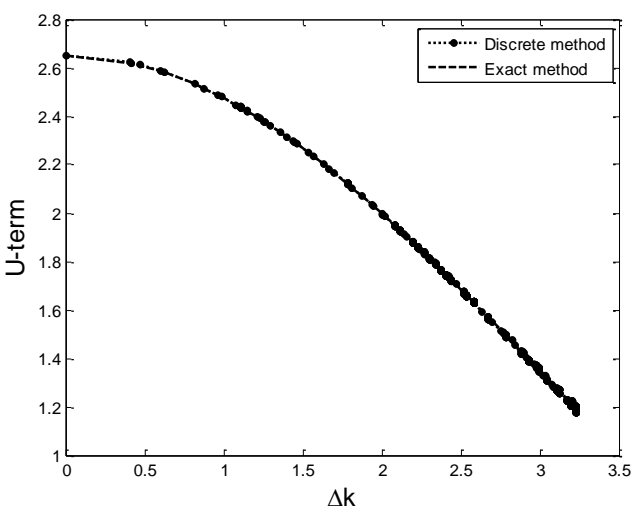

Fig. 8. Comparison of first Born term using the Holt and the Discrete (with 48 internal wavectors) methods. 
Moreover, an analysis of the consequence of the discretization of the volume has been carried out. The stair-casing error has been computer for different number of cells used to discretize the scattering particle volume. Fig. 9 shows that as the goemetrical discretization is refined further and further, the star-casing error tends to zero.

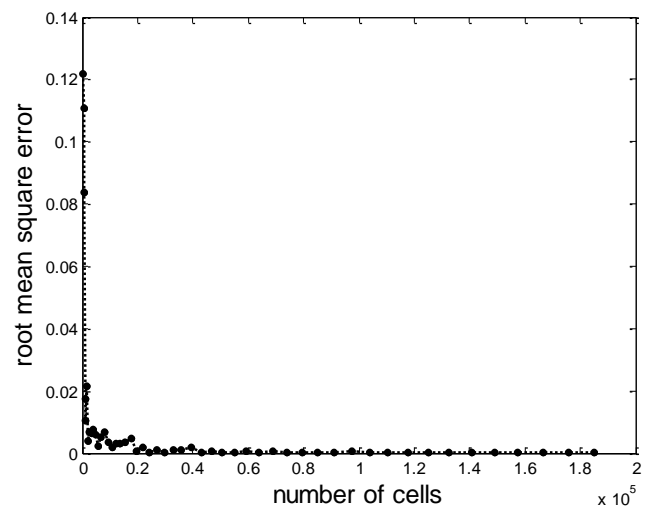

Fig. 9. The plot of root mean square error of U-terms agaianst number of cells.

\section{B. Scattering by spherical particles}

Spheres have been used more extensively in scattering problems than any other particle shape. This is partly because it is the only three dimensional particle for which an analytical closed form solution is available and therefore can be easily used to validate new methods.

To validate the scattering amplitude, we consider an incident plane wave linearly polarized propagating in the $+z$ direction. We compare our method with the established Mie theory as implemented in [37]. The $\mathrm{C}$ frequency band $(5.8 \mathrm{GHz})$ is selected to suit the operating frequency of European weather radar, giving a wavenumber $k_{0} \approx 1 \mathrm{~cm}^{-1}$. For comparison with the Mie theory, the refractive index $n=1.33$ has been selected as in [38, p.153].

We perform the comparison between the scattering cross section evaluated with the Discrete Method and with the Mie theory for different size parameter values.

The normalised differential scattering cross section for a size parameter $x=0.1$ is shown in Fig. 10. For the selected frequency, $x=0.1$ corresponds to a particle of diameter $d \approx 0.15$ $\mathrm{cm}$. The forward and backward ratio for both calculation by the Mie theory (Rayleigh regime) and the Discrete Method are virtually identical for the horizontal polarization and similar for the vertical polarization. The forward to backward ratio is approximately 1 as expected.

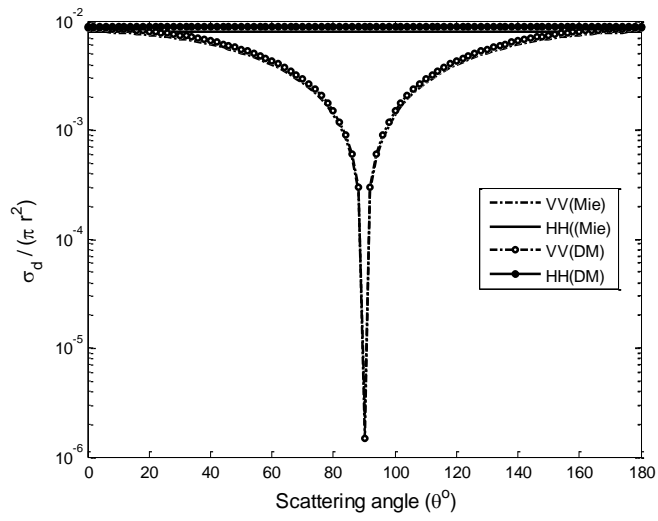

Fig. 10. Normalised differential scattering cross section computed with the Discrete Method and with the Rayleigh theory from a non-absorbing dielectric sphere of size parameter $x=0.1$ and $n=1.33$ for an input wave of $f=5.8$ $\mathrm{GHz}$.

The normalised differential scattering cross section for the size parameter $x=0.5$ is shown in Fig. 11. In this case, the particle diameter is $d \approx 0.8 \mathrm{~cm}$. From the figure it is clear that the curves follow a similar pattern as expected but the forward and backward scattering ratio have increased to more than 1. This is due to the phase shift as the particle size tends towards the Mie regime.

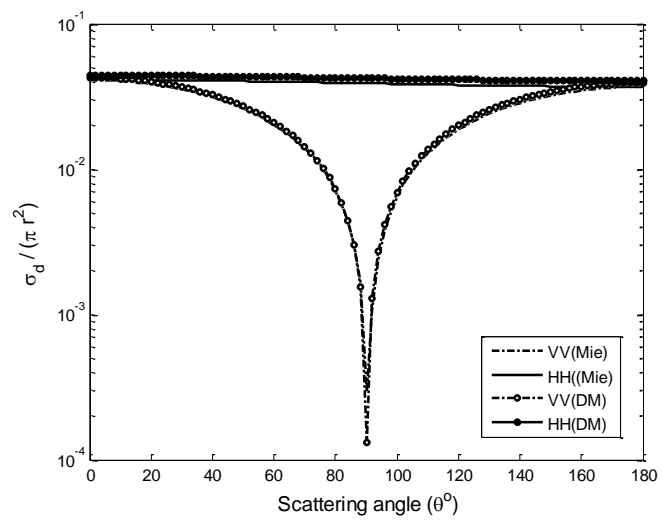

Fig. 11. Normalised differential scattering cross section computed with the Discrete Method and with the Mie theory (Rayleigh regime) from a nonabsorbing dielectric sphere of size parameter $x=0.5$ and $n=1.33$ for an input wave of $f=5.8 \mathrm{GHz}$.

The normalised differential scattering cross section for the size parameter $x=1$ is shown in Fig. 12. In this case, the particle diameter is $d \approx 2.0 \mathrm{~cm}$ which is approximately the characteristic length of a large ice aggregate [39], [40]. The numerical results show good agreement. 


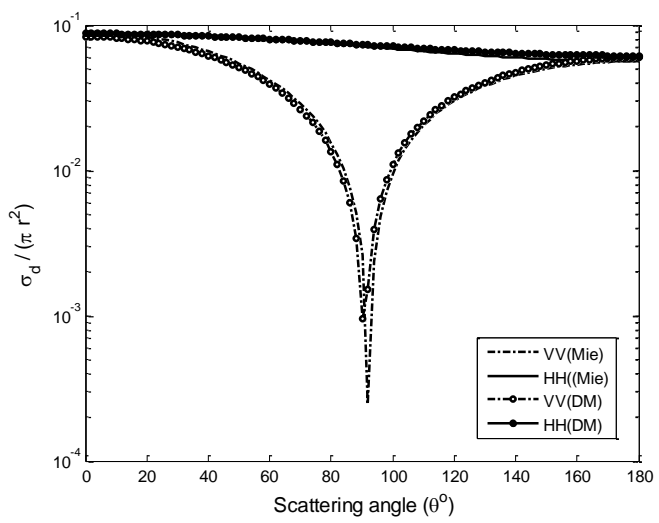

Fig. 12. Normalised differential scattering cross section computed with the Discrete Method and with the Rayleigh theory from a non-absorbing dielectric sphere of size parameter $x=1$ and $n=1.33$ for an input wave of $f=5.8$ $\mathrm{GHz}$.

In the Mie regime for spherical particles both polarizations must have the same magnitude at the forward scattering function based on the symmetry [38]. As the size parameter $x$ increases, it is expected that more complex interactions will occur between the scatterer and the plane wave. This will result in a change of the curves for both polarizations with more peaks and valleys along the scattering angle. Further tests have been made including compliance with the law of reciprocity and the angular dependency were satisfactory.

\section{SCATTERING BY MIXED PHASE HYDROMETEORS}

After satisfactory results have been obtained for homogeneous particles in the Mie regime, we attempt to evaluate the scattering amplitude function from hybrid hydrometeors modelled as inhomogeneous dielectric particles using the Discrete Method. In particular, melting snowflake aggregates containing air, ice and liquid water are good representation of such scatterers with mixed dielectric constants. However, in this paper dry snowflakes which are a mixture of air and ice are considered. The scattering cross section of dry snowflakes is evaluated firstly using mixing theories comparing the results of the DM with the Mie theory. Additionally, we compare the scattering cross section computed with the DM for a dry snowflake modelled as a discretised inhomogeneous particle and modelled as a inhomogeneous particle with effective dielectric constant evaluated with the Maxwell Garnett rule.

Firstly, we start by applying Maxwell Garnett two-part mixing formula discussed in [6] to dry snowflakes that we model as spherical particles. When using effective medium theories with separated topology like the Maxwell Garnett to estimate effective dielectric constant, considerations must be made as to which component will be the inclusion and which the matrix. Significant differences appear in the effective dielectric constant if the two materials are simply interchanged. For this reason, we decide to use a volume of $50 \%$ for both components. In [41] to represent non-absorbing ice crystals, a refractive index $n_{\text {ice }}=1.78$ is chosen to be the host or background material and $n_{\text {air }}=1.0$ to be the inclusion or guest. The effective dielectric constant is obtained to be approximately $\varepsilon_{\text {mix }} \approx 1.839$ corresponding to $n_{\text {mix }} \approx 1.356$.
The size parameter is chosen to be $x=1$ and the frequency $5.8 \mathrm{GHz}$.

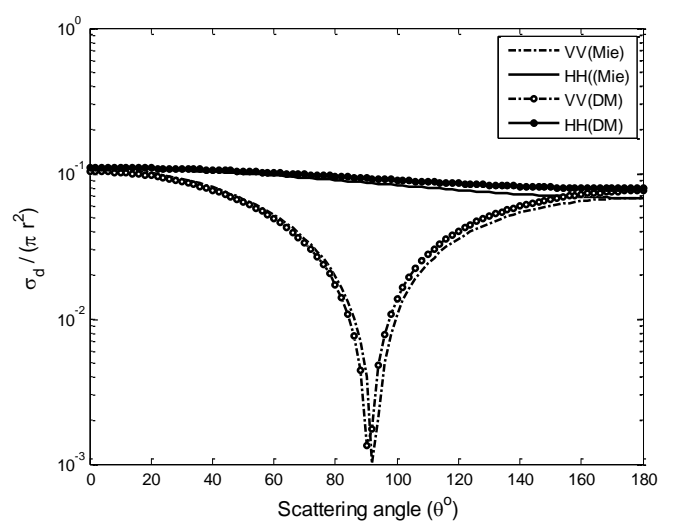

Fig. 13. Normalised differential scattering cross section computed with the Discrete Method and with the Rayleigh theory from a non-absorbing dielectric sphere using Maxwell-Garnett formula for air inclusions in an ice matrix (iceair mixture) given $x=1.0$ and $n_{\text {mix }} \approx 1.356$.

The normalized differential scattering cross section computed with the Mie theory and the Discrete Method for dry snowflakes using the effective dielectric constant are shown in Fig. 13. Remarkably, the curves are in good agreement. The forward and backward ratio in both cases converges reasonably. The amplitude of the forward scattering falls off gradually due to phase shift at the backward direction for the case of the horizontal polarization. In order to investigate the validity of effective medium theories to evaluate scattering properties from inhomogeneous particles we use the DM for dry snowflakes modelled as

- homogeneous particles of effective dielectric constant evaluated with the Maxwell Garnett rule

- discretised inhomogeneous particles

assuming both air and ice with volume fractions of $50 \%$. In the discretised case the scatterer is modelled as a sphere of cells where half of them has been assigned the dielectric constant $n_{\text {ice }}=1.78$ and the other half $n_{\text {air }}=1$ using a uniform distribution spatially. Again, the size parameter is chosen to be $x=1$ and the frequency $5.8 \mathrm{GHz}$ as previously. Fig. 14 shows the normalized differential scattering cross section computed using the Discrete Method for dry snowflakes modelled with the Maxwell Garnett mixing formula and without effective medium theory as a sphere of cells of different refractive indices. 


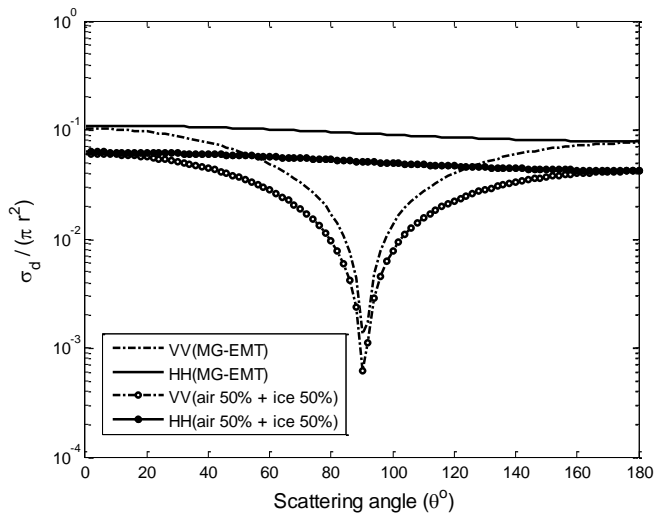

Fig. 14. Normalised differential scattering cross section computed with the Discrete Method from a non-absorbing dry snowflake of size parameter $x=1$ using Maxwell-Garnett formula with an effective dielectric constant $\varepsilon_{\text {mix }} \approx$ 1.839 and without mixing theories modelling the snowflake as a sphere of cells of different refractive indices ( $n_{\text {air }}=1$ and $\left.n_{\text {ice }}=1.78\right)$.

Although the curves shown in Fig. 14 follow similar pattern, they significantly deviate. The reason for this difference has been identified in how the calculations of the Z-term and Wterm scale in the homogeneous and inhomogeneous case.

For dry snowflakes as a equal mixture of ice $n_{\text {ice }} \approx 1.78$ and air $n_{\text {air }} \approx 1$, the effective refractive index computed using Maxwell-Garnett formula is $n_{\mathrm{mix}} \approx 1.356$. The correspondent permittivities are then given as

$$
\varepsilon_{r}^{\text {ice }} \approx 3.168 \quad \text { and } \quad \varepsilon_{r}^{\text {mix }} \approx 1.839 .
$$

Using the discretization approach for dry snowflakes, having half of the cells filled with ice, their relative density is $\frac{1}{2}$ so in the W-term (22) the contribution of $\gamma\left(\mathbf{r}_{i}\right) \varepsilon\left(\mathbf{r}_{i}\right)=$ $k_{0} \varepsilon\left(\mathbf{r}_{i}\right)\left(\varepsilon\left(\mathbf{r}_{i}\right)-1\right)$ for all the cells reduces to

$$
\frac{\varepsilon_{r}^{\text {ice }}\left(\varepsilon_{r}^{\text {ice }}-1\right)}{2}=\frac{3.168 \times 2.168}{2}=3.434 .
$$

Using the effective medium theory, we have $100 \%$ fill, so the contribution amounts to

$$
\varepsilon_{r}^{\operatorname{mix}}\left(\varepsilon_{r}^{\operatorname{mix}}-1\right)=1.839 \times 0.839=1.543 .
$$

Similarly for the Z-term, the relative density of the cell pairs for the U-terms amounts to $1 / 4$, hence the Z-term is scaled with

$$
\frac{1}{4}\left(\varepsilon_{r}^{\text {ice }}-1\right)^{2}=\frac{(2.168)^{2}}{4}=1.175,
$$

and for the effective medium, all cell pairs count, but with smaller polarizabilities $\gamma$, so $Z$ is scaled with

$$
\left(\varepsilon_{r}^{\operatorname{mix}}-1\right)^{2}=0.839^{2}=0.704 .
$$

The W-term, the Z-term and consequently the $\mathrm{K}$-term are relatively smaller for the effective medium theory approach so the inversion of the equations will give rise to larger amplitudes for the Fourier coefficients $\overline{\mathbf{C}}_{m}$ (47). The scaling of the U-term is neutral because it is a factor on both sides for the forward and substitution steps.

The approach of using effective medium theory such as two parts Maxwell Garnett to estimate dielectric constant of the inhomogeneous medium is shown to produce an inaccurate result for mixed phase hydrometeors. This result shows that mixing two media is not a linear process as it is assumed in effective medium theories.

\section{CONClusion}

In this work we have introduced our new approach, the Discrete Method which provides an efficient technique to evaluate the scattering properties by irregular inhomogeneous dielectric particles without using effective medium theories. The key feature of this method is that the evaluation of the scattering properties is computationally fast in both implementation and execution. For inhomogeneous particles composed by two media such as dry snowflakes modelled with effective medium theories, the DM compares well with Mie theory. However, the DM shows that using mixing rules leads to an overestimation of the scattering cross section. This is due to the fact that mixing media is not a linear process as it is assumed. Since in this work only the first degree of the expansion of the Z-term is considered, the evaluation of the scattering properties presented here is adequate for size parameter up to 1 . Our method also has the potential to be applied to an ensemble collection of hydrometeors. In this case, the best way to carry out the computation would be to evaluate K-term for a suitably densely sampled set and carry out the inversion for each scattering geometry as required.

\section{ACKNOWLEDGMENT}

David Bebbington would like to acknowledge a debt to the late Anthony Holt for his inspirational insigths into scattering theory. Finally, we would also like to thank the anonymous reviewers for their valuable comments and suggestions to improve the quality of the paper.

\section{REFERENCES}

[1] L. Liao and R. Meneghini, "On modeling air/spaceborne radar returns in the melting layer," Geoscience and Remote Sensing, IEEE Transactions on, vol. 43, no. 12, pp. 2799-2809, 2005.

[2] R. Meneghini and L. Liao, "Effective dielectric constants of mixedphase hydrometeors," Journal of Atmospheric and Oceanic Technology, vol. 17, no. 5, pp. 628-640, 2000.

[3] J. Steinert and M. Chandra, "Melting-layer modelling at C-band," Advances in Radio Science, vol. 8, no. 16, pp. 285-288, 2010.

[4] F. Fabry and W. Szyrmer, "Modeling of the melting layer. Part II: Electromagnetic," Journal of the Atmospheric Sciences, vol. 56, no. 20, pp. 3593-3600, 1999.

[5] T. H. Stein, C. D. Westbrook, and J. Nicol, "Fractal geometry of aggregate snowflakes revealed by triple-wavelength radar measurements," Geophysical Research Letters, vol. 42, no. 1, pp. 176-183, 2015.

[6] J. C. Maxwell-Garnett, "Colours in metal glasses and in metallic films." Proceedings of the Royal Society of London, vol. 73, no. 488-496, pp. 443-445, 1904.

[7] V. D. Bruggeman, "Berechnung verschiedener physikalischer Konstanten von heterogenen Substanzen. I. Dielektrizitätskonstanten und Leitfähigkeiten der Mischkörper aus isotropen Substanzen," Annalen der Physik, vol. 416, no. 7, pp. 636-664, 1935.

[8] M. I. Mishchenko, Z. M. Dlugach, and N. T. Zakharova, "Direct demonstration of the concept of unrestricted effective-medium approximation," Optics letters, vol. 39, no. 13, pp. 3935-3938, 2014.

[9] C. Liu, R. L. Panetta, and P. Yang, "Inhomogeneity structure and the applicability of effective medium approximations in calculating light scattering by inhomogeneous particles," Journal of Quantitative Spectroscopy and Radiative Transfer, vol. 146, pp. 331-348, 2014. 
[10] M. Kahnert, "Modelling radiometric properties of inhomogeneous mineral dust particles: Applicability and limitations of effective medium theories," Journal of Quantitative Spectroscopy and Radiative Transfer, vol. 152, pp. 16-27, 2015.

[11] M. I. Mishchenko, J. M. Dlugach, and L. Liu, "Applicability of the effective-medium approximation to heterogeneous aerosol particles," Journal of Quantitative Spectroscopy and Radiative Transfer, vol. 178, pp. 284-294, 2016.

[12] M. I. Mishchenko, J. M. Dlugach, M. A. Yurkin, L. Bi, B. Cairns, L. Liu, R. L. Panetta, L. D. Travis, P. Yang, and N. T. Zakharova, "First-principles modeling of electromagnetic scattering by discrete and discretely heterogeneous random media," Physics Reports, vol. 632, pp. $1-75,2016$.

[13] B. K. Wilson, M. R. Behrend, M. P. Horning, and M. C. Hegg, "Detection of malarial byproduct hemozoin utilizing its unique scattering properties," Optics Express, vol. 19, no. 13, pp. 12 190-12 196, 2011.

[14] H. Lindner, G. Fritz, and O. Glatter, "Measurements on concentrated oil in water emulsions using static light scattering," Journal of Colloid and Interface Science, vol. 242, no. 1, pp. 239-246, 2001.

[15] T. Wriedt, "Mie theory: A review," in The Mie Theory. Springer, 2012, pp. 53-71.

[16] C. F. Bohren and D. R. Huffman, Absorption and scattering of light by small particles. Wiley. com, 2008.

[17] B. T. Draine, "The discrete-dipole approximation and its application to interstellar graphite grains," The Astrophysical Journal, vol. 333, pp. 848-872, 1988.

[18] P. J. Flatau and B. Draine, "Discrete-dipole approximation for scattering calculations," J. Opt. Soc. Am. A, vol. 11, p. 1491, 1994.

[19] A. Holt, N. Uzunoglu, and B. Evans, "An integral equation solution to the scattering of electromagnetic radiation by dielectric spheroids and ellipsoids," Antennas and Propagation, IEEE Transactions on, vol. 26, no. 5, pp. 706-712, 1978.

[20] N. Uzunoglu, B. Evans, and A. Holt, "Evaluation of the scattering of an electromagnetic wave from precipitation particles by the use of Fredholm integral equations," Electronics Letters, vol. 12, no. 12, pp. 312-313, 1976.

[21] A. Holt, "The fredholm integral equation method and comparison with the t-matrix approach," in Acoustic, Electromagnetic and Elastic Wave Scattering-Focus on the T-Matrix Approach, 1980, pp. 255-268.

[22] J. Avelin, R. Sharma, I. Hanninen, and A. H. Sihvola, "Polarizability analysis of cubical and square-shaped dielectric scatterers," IEEE Transactions on Antennas and Propagation, vol. 49, no. 3, pp. 451-457, 2001.

[23] J. Helsing and K.-M. Perfekt, "On the polarizability and capacitance of the cube," Applied and Computational Harmonic Analysis, vol. 34, no. 3, pp. 445-468, 2013.

[24] F. O. Ngobigha, "Development of a discrete numerical approach to the Fredholm Integral Method for evaluating microwave scattering by irregular hydrometeors." Ph.D. dissertation, University of Essex, Dec. 2015.

[25] R. G. Newton, Scattering theory of waves and particles. DoverPublications. com, 1982.

[26] M. I. Mishchenko, Electromagnetic scattering by particles and particle groups: An introduction. Cambridge University Press, 2014.

[27] M. L. Boas, Mathematical methods in the physical sciences. Wiley, 2006.

[28] R. Snieder and K. Van Wijk, A guided tour of mathematical methods for the physical sciences. Cambridge University Press, 2004.

[29] N. K. Uzunoglu, "Theoretical calculation of scattering of electromagnetic waves from precipitation particles." Ph.D. dissertation, University of Essex, 1976.

[30] A. Holt and B. Santoso, "The Fredholm integral method. II. The calculation of scattering amplitudes for potential scattering," Journal of Physics B: Atomic and Molecular Physics, vol. 6, no. 10, p. 2010, 1973.

[31] G. N. Watson, A treatise on the theory of Bessel functions. Cambridge university press, 1995.

[32] C. Schwartz, "A class of discontinuous integrals involving bessel functions," Journal of Mathematical Physics, vol. 23, no. 12, pp. 2266-2267, 1982.

[33] F. O. Ngobigha and D. H. O. Bebbington, "Electromagnetic waves scattering by dielectric ellipsoids applying integral equation approach," in Radio Science Meeting (Joint with AP-S Symposium), 2014 USNCURSI. IEEE, 2014, pp. 227-227.

[34] J. G. Van Bladel, Electromagnetic fields. John Wiley \& Sons, 2007, vol. 19.
[35] W. Zhang, J. K. Tervonen, and E. T. Salonen, "Backward and forward scattering by the melting layer composed of spheroidal hydrometeors at 5-100 GHz," Antennas and Propagation, IEEE Transactions on, vol. 44, no. 9, pp. 1208-1219, 1996.

[36] S. Y. Matrosov, "Assessment of radar signal attenuation caused by the melting hydrometeor layer," Geoscience and Remote Sensing, IEEE Transactions on, vol. 46, no. 4, pp. 1039-1047, 2008.

[37] P. W. Barber and S. C. Hill, Light scattering by particles: Computational methods. World scientific, 1990, vol. 2.

[38] H. C. Hulst and H. C. van de Hulst, Light scattering by small particles. Courier Dover Publications, 1957.

[39] C. Schmitt and A. Heymsfield, "The dimensional characteristics of ice crystal aggregates from fractal geometry," Journal of the Atmospheric Sciences, vol. 67, no. 5, pp. 1605-1616, 2010.

[40] C. Westbrook, R. Ball, and P. Field, "Radar scattering by aggregate snowflakes," Quarterly Journal of the Royal Meteorological Society, vol. 132, no. 616, pp. 897-914, 2006.

[41] M. N. Sadiku, "Refractive index of snow at microwave frequencies," Applied Optics, vol. 24, no. 4, pp. 572-575, 1985.

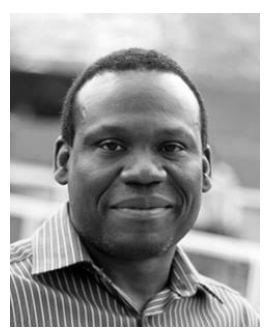

Felix Ngobigha received the BTech. degree in electrical engineering from the Rivers State University of Science \& Technology, Nigeria, in 2001, and the MSc. and Ph.D. degrees in telecommunications, computing and electronic systems from University of Essex, U.K., in 2005 and 2015, respectively. From 2001 to 2004, he was with Centre for Satellite Technology Development, Nigeria as a Scientific Officer. He has held lecturing position at Niger Delta University, Nigeria, for several years. Since 2016 he has been with the School of Computer Science and Electronic Engineering, University of Essex, U.K. His research interests focus on wave propagation and scattering for remote sensing, microstrip antenna design, and wireless communications.

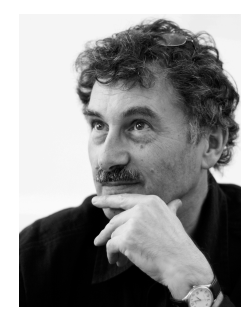

David Bebbington received the B.A. degree in experimental and theoretical physics and the Ph.D. degree in radio astronomy from Cambridge University, Cambridge, U.K., in 1977 and 1986, respectively. From 1981 to 1984 , he worked on millimeter wave propagation research at the Rutherford Appleton Laboratory. Since 1984, he has been at the University of Essex, Essex, U.K., and currently holds the post of Senior Lecturer in the School of Computer Science and Electronic Engineering. His interests are polarimetry, scattering, applications of wave propagation in remote sensing, and weather radars.

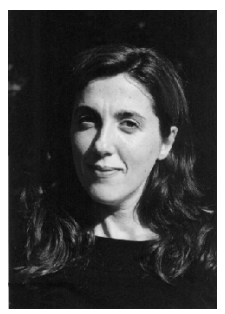

Laura Carrea received the Laurea degree in Physics from the University of Turin, Italy in 1997 and her $\mathrm{Ph} . \mathrm{D}$. in Electronic Engineering from the University of Essex, U.K. in 2013. From 1999 to 2004 she was with the Chemnitz University of Technology, Germany partly with a Fellowship in the frame of the Marie Curie TMR Network "Radar Polarimetry: Theory and Application". From 2004 to 2013 she was with Centre for Remote Sensing \& Environmetrics at the University of Essex, U.K. and from 2014 with the Meteorology Dept. at the University of Reading, U.K. Her main interests include polarization and scattering for remote sensing and surface temperature retrieval from satellite for climate studies. 